\title{
CORSIKA Based Simulations of Background in Baikal Experiment
}

\author{
Blahoslav Pastirčák ${ }^{1, \star}$ \\ ${ }^{1}$ Institute of Experimental Physics of Slovak Academy of Sciences, Košice, Slovak republic
}

\begin{abstract}
The Baikal experiment aims to register cosmological neutrinos and map the high-energy neutrino sky in the Southern Hemisphere including the region of the Galactic Center. It will use a $\mathrm{km}^{3}$-scale high-energy neutrino telescope located in the southern basin of the Lake Baikal. The northern location of the detector site allows direct observation of the Galactic Center in more than $75 \%$ of the astronomical time. The selection of events from neutrino induced upward going muons, suggests a fairly reliable estimation of the expected background from atmospheric muons. The procedure for simulating background events from atmospheric muons in an array is performed in several steps. The CORSIKA 7.64 was used to simulate the flux of atmospheric muons at the sea level with appropriate chemical composition of the primary cosmic rays. The muon propagation through water and rock to the array level was then simulated with the MUM code. As the last step of the simulation chain, the detector response to the Cherenkov radiation of muons was estimated by taking into account the features of the array measuring systems was performed. The main features of the CORSIKA Monte Carlo code and the next steps of the simulation chain are summarized. The physical models embedded in CORSIKA are described. Application of the full simulation chain is demonstrated.
\end{abstract}

\section{Introduction}

The Baikal experiment is one of the three deep underwater experiments using neutrino telescopes to detect high-energy neutrinos. The Baikal collaboration has created years ago the first generation underwater neutrino telescope NT200 which demonstrated the possibility to register cosmic neutrinos by such a technique. At present, the next-generation neutrino telescope Baikal-GVD of $\mathrm{km}^{3}$ scale is built at the same site [1]. It aims at detecting cosmological neutrinos and subsequently discover and study individual Galactic and Extragalactic neutrino "point sources" in the energy range $E>\mathrm{TeV}$. Further physics goals are to investigate diffuse neutrino flux - energy spectra, local and global anisotropy, flavor content, transient sources $(\mathrm{GRB}, \ldots)$, dark matter - indirect search, exotic particles - monopoles, Q-balls. The related IceCube neutrino telescope has already registered cosmological neutrinos [2]. While IceCube efficiently observes the Northern sky, Baikal-GVD and KM3NeT [3] will have a better coverage of the Southern sky with one of the most interesting objects, the Galactic Center, and will be complementary to the IceCube. The plan for Baikal-GVD present stage (up to 2020-2021) is a facility consisting of 2304 optical modules with an effective detection volume of $0.4 \mathrm{~km}^{3}$ for cascades

^e-mail: slavo@saske.sk 
with $E>100 \mathrm{TeV}$. At the next stage, this volume will be increased to $1.5 \mathrm{~km}^{3}$. Very precise and detailed simulations of the background for such rare events that Baikal has the ambition to register is necessary.

\section{Baikal experiment}

The principle of the high-energy neutrino detection is to register Cherenkov radiation emitted by secondary charged particles created in neutrino interactions. Because of the very rare interaction rates, a detector must reach the Gigaton scale in order to efficiently detect cosmic neutrinos. Such a telescope is a large scale Cherenkov detector consisting of a 3D array of ultra-sensitive photosensors deployed deep underwater in order to significantly decrease the atmospheric muon background as well as to fully suppress the sunlight. The setup of the Baikal-GVD together with the geographical coordinates of the detector site are shown in Figure 1.
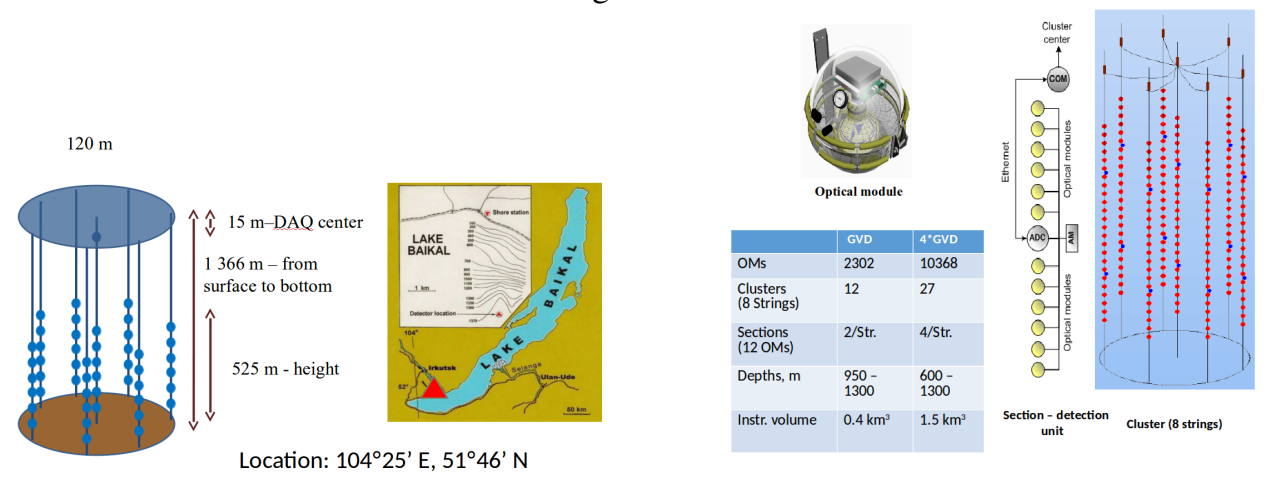

Figure 1. (Color online) Baikal-GVD essentials. The photosensor and the readout electronics are housed in a pressure-resistant glass sphere, the so called optical module (OM) [4]. The OMs are distributed along flexible strings. One end of each string is anchored to the lake floor while the other end is held in almost vertical position by a buoy $25 \mathrm{~m}$ below the lake surface. There are $36 \mathrm{OMs}$ on one string, spaced by $15 \mathrm{~m}$. The concept of strings is modular by design. A collection of 8 strings forms a single separate building block called a cluster.

\section{Monte Carlo simulation of the background in Baikal}

The main goal of the Monte Carlo (MC) simulations is to characterize the muon tracks produced by the flow of astrophysical neutrinos in the Earth atmosphere, and to distinguishing them from the background produced by other sources in the Baikal experiments. The vast majority of events in the detector occurs from atmospheric muon bundles. Background sources are the atmospheric muons, atmospheric muon neutrinos, atmospheric electron neutrinos. ANIS [5] is the high energy MC event generator for neutrino telescopes. The simulation of the contributions coming from the atmospheric muons is done by means full simulation chain which is based on the CORSIKA program [6]. The muons from the cosmic-ray-induced showers reaching the sea-surface are then propagated through water and rock to the detector simulated with the MUM (MUons + Medium) code [7]. In this way, it is possible to evaluate the response of the detector to a given cosmic-ray flux. It is performed in several steps as described in Figure 2.

Our present simulations are based on the former ones for NT200, where the CORSIKA 5.7 code [4] was used to simulate the flux of atmospheric muons at sea level, with appropriate chemical composition of the primary cosmic rays. 


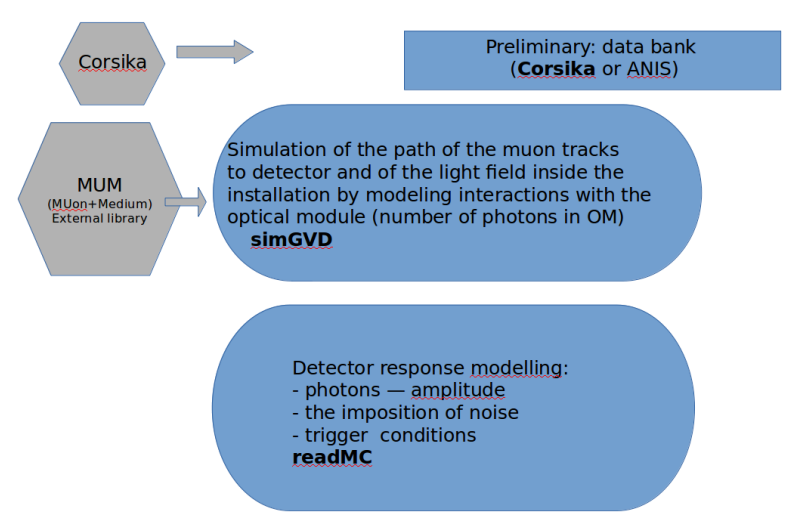

Figure 2. Simulation chain: 1. Modelling the atmospheric muons flux at the sea level; 2. Simulation of the interactions with media producing secondary muons; 3 . Muon propagation in water and transport up to detector; 4. Simulation of the detector response to Cherenkov radiation including electronic system performance

\section{CORSIKA}

The first step of the simulation chain is the simulation of the primary muon transport through the Earth's atmosphere up to the sea level. It is performed by widely used extensive air showers (EAS). The MC code CORSIKA, provides the most realistic EAS taking into account several different hadronic interaction models. CORSIKA may be used up to and beyond energies of $100 \mathrm{EeV}$. It consists basically of four parts:

- a general frame handling the $\mathrm{I} / \mathrm{O}$, performing decay of unstable particles and tracking particles assuming ionization energy loss and deflection by multiple scattering and the Earth magnetic field;

- treatment of the hadronic interactions of nuclei and hadrons with air nuclei at higher energies;

- simulation of the hadronic interactions at lower energies;

- description of transport and interaction of electrons, positrons and photons.

The main source of uncertainty in the prediction of shower observables for different primary particles and energies is currently dominated by the differences among the hadronic interaction models. CORSIKA uses 6 high-energy models - DPMJET [8], EPOS [9], NEXUS, QGSJET [10], SIBYLL [11], VENUS [12] and 3 low-energy ones - FLUKA [13], GHEISHA, UrQMD [14].

GHEISHA and NEXUS are obsolete nowadays (however GHEISHA still widely used). New accelerator data together with more precise air shower experiments started major new activities in hadronic modelling resulting in new generation interaction models released in CORSIKA. Currently three main hadronic models are intensively maintained: EPOS-LHC, QGSJET II 04 and SIBYLL 2.3. They are in good agreement with the LHC data and provide reasonably good description of inclusive shower observables.

\subsection{The present Baikal-GVD MC simulation chain based on CORSIKA 7}

For the present simulations of the muon background in the GVD detector, a new version of CORSIKA, CORSIKA 7 (versions 7.64-7.71), was used as the basis for the subsequent full simulation chain. This version was significantly improved as compared to CORSIKA 5 mainly due to the availability of LHC data and taking into account new precise astroparticle measurements. CORSIKA simulations with relevant high energy hadronic models (as described above) were performed and compared. The low energy model is less important in our case, we have used UrQMD and FLUKA. Several special input parameters like curved version to cover full zenith angle, upward version for upward going particles, slant depth for longitudinal distributions and particle output written in compact form have 
been selected.The energy of primaries cover the range from $240 \mathrm{Gev}$ up to $100 \mathrm{PeV}$. The simulations have been performed for the following fixed chemical composition of the primaries: p, He, CNO, Fe. The utility for merging these samples (optionally with weights) was prepared and used to create a databank from CORSIKA 7 simulations, which will be used in the next simulation steps. At present we have over $10^{9}$ events in this databank $\left(1.1 \cdot 10^{9} \mathrm{p}, 4.0 \cdot 10^{8} \mathrm{He} .4 .3 \cdot 10^{7} \mathrm{CNO}\right.$ and $7.3 \cdot 10^{6} \mathrm{Fe}$ events).

For the propagation of muons in water the above mentioned Fortran based MUM package will still be used, but at the same time the $\mathrm{C}++$ based package PROPOSAL [15] was implemented. It is public $\mathrm{MC}$ tool for charged lepton propagation through media. The code is capable of propagating muons of energies from muon rest mass and tau leptons of energies from tau rest mass to $10^{11} \mathrm{GeV}$ or higher, is fast and precise. The immediate task is to compare the results obtained by this two codes.

\subsection{Next generation CORSIKA}

In 2018, the CORSIKA core team started the development of the completely new program CORSIKA 8 written in $\mathrm{C}++$ with modular design. It will support future astroparticle physics experiments with solid foundation for simulations and research.

The main advantage of the new CORSIKA for Baikal and other neutrino telescopes (ICeCube, Antares, KM3Net) is that the same Cherenkov physics, where Cherenkov emission from single particles and small showers in deep water or ice, needs to be tracked through the medium to the detector. There could be a general large benefit, if the Cherenkov tracking code could be made available in the best possible implementation as part of CORSIKA for the whole community.

\section{Summary}

We have prepared software for MC simulations of the background in Baikal experiment based on CORSIKA package in the following steps:

- Upgrade to CORSIKA 7 simulations for Baikal-GVD, comparison of models, their selection optimization and creating a CORSIKA 7 databank.

- Using PROPOSAL besides MUM in the simulation steps following CORSIKA.

- Coordination with CORSIKA 8 development team for the next generation CORSIKA.

\section{References}

[1] A.D. Avrorin, et al., EPJ Web Conf. 116, 01003 (2016)

[2] M.G. Artsen, et al., Science 342, 1242856 (2013)

[3] S. Adrian-Martinez, et al., J. Phys. G: Nucl. Part. Phys 43, 084001 (2016)

[4] L. Capdevielle, et al., KfK Report4998 Karksruhe: Kernforschungzentrum (1992)

[5] A.Gazizov, M.Kowalski, Computer Physics Communications 172, 203 (2005)

[6] D. Heck, J. Knapp, Forschungzentrum Karlsruhe Report FZKA 6097 (1998)

[7] I.A. Sokalsky, et al., Phys. Rev. D 64, 074015 (2001)

[8] J. Ranft, et al., Phys. Rev. D 51, 64 (1995)

[9] K. Werner, et al., Phys. Rev. C 74, 044902 (2006)

[10] S.S. Ostapchenko, Phys. Rev. D 74, 014026 (2006)

[11] E.J. Ahn, R. Engel, T.K. Gaisser, P. Lipari, T. Stanev, Phys. Rev. D 80, 094003 (2009)

[12] K. Werner, Phys. Rep. 232, 87 (1993)

[13] A. Ferrari, P. Sala, A. Fasso, J. Ranft, CERN-2005-10, INFN/TC 05/11, SLAC-R-773 (2005)

[14] M. Bleicher, et al., J. Phys. G: Nucl. Part. Phys. 25, 1859 (1999)

[15] M. Dunsch, et al., Computer Physics Communications 242, 132 (2019) 